\title{
Monte Carlo Calculations of Selected Dose Components in a Head Model for Boron Neutron Capture Therapy
}

\author{
Institute of Atomic Energy, 05-400 Otwock-Świerk, Poland \\ e-mail: kasia.tyminska@gmail.com
}

\begin{abstract}
Boron Neutron Capture Therapy is a very promising form of cancer therapy, consisting in irradiating a stable isotope of boron $\left({ }^{10} \mathrm{~B}\right)$ concentrated in tumor cells with a low energy neutron beam. This technique makes it possible to destroy tumor cells, leaving healthy tissues practically unaffected. In order to carry out the therapy in the proper way, the proper range of the neutron beam energy has to be chosen.

In this paper we present the results of the calculations, using the MCNP code, aiming at studying the energetic dependence of the absorbed dose from the neutron capture reaction on boron (the therapeutic dose), and hydrogen and nitrogen (the injuring dose).
\end{abstract}

Key words: BNCT, boron neutron capture therapy.

\section{Introduction}

BNCT (Boron Neutron Capture Therapy) is a form of radiotherapy that held promise of being beneficial to cancer patients with glioma, a type of brain cancer. This type of tumor is quite resistant to other treatment modalities and the prospects of its cure are very poor.

An ideal therapy for cancer would be one whereby all tumor cells were selectively destroyed without damaging normal tissues.

BNCT brings together two components that, when kept separate, have only minor effects on cells. The first component is a stable isotope of boron (boron-10) that can be 
concentrated in tumor cells by being attached to tumor-seeking compounds. The second one is a beam of low-energy neutrons. Boron-10 in or adjacent to tumor cells disintegrates after capturing a neutron. The high-energy, heavy charged particles produced destroy only the cells, primarily cancer cells, in close proximity to the vertex, leaving adjacent normal cells largely unaffected.

The dose absorbed by the tissue in BNCT consists of four components:

1. The gamma ray dose from the gamma ray component of the beam and the gamma rays induced in the tissue, mainly due to ${ }^{1} \mathrm{H}(\mathrm{n}, \gamma)^{2} \mathrm{H}$ reactions emitting $2.2 \mathrm{MeV}$ gamma rays.

2. The fast neutron dose epithermal and fast neutrons that cause "knock-out" recoil protons from hydrogen in tissue.

3. The nitrogen dose from ${ }^{14} \mathrm{~N}$ absorbing a thermal neutron and emitting $0.6 \mathrm{MeV}$ a proton in ${ }^{14} \mathrm{~N}(\mathrm{n}, \mathrm{p}){ }^{14} \mathrm{C}$ reaction.

4. The boron dose from ${ }^{10} \mathrm{~B}$ absorbing a thermal neutron in a ${ }^{10} \mathrm{~B}(\mathrm{n}, \alpha){ }^{7} \mathrm{Li}$ reaction. The emitted $\alpha$ particle and the recoiling ${ }^{7} \mathrm{Li}$ ion result in locally deposited energy, averaging about $2.34 \mathrm{MeV}$. In $94 \%$ of the reactions the recoiling ${ }^{7} \mathrm{Li}$ ion is produced in an excited state and de-excites emitting a $477 \mathrm{keV}$ gamma ray. In the remaining $6 \%$ of events, ${ }^{7} \mathrm{Li}$ ion is emitted in the ground state.

The only usable component from the therapeutic point of view is the boron component, therefore we want the boron dose to be as large as possible while other components are kept minimal. Among other contributions of the absorbed dose, those most significant from the dosimetry perspective are the nitrogen dose and the $2.2 \mathrm{MeV}$ gamma dose from the hydrogen capture.

If the tumor tissue is situated in the brain at a depth of about 2-3 cm, the most usable neutrons are those epithermal ones with energies of $0.5 \mathrm{eV}-10 \mathrm{keV}$. They are moderated in the tissue between the skin and the tumor cells and they gain thermal energies (below $0.5 \mathrm{eV}$ ), when reaching the tumor tissue (the cross-section for the reaction with ${ }^{10} \mathrm{~B}$ is inversely proportional to neutron velocity). Direct irradiation with a thermal neutron beam would cause a big irradiation of the healthy tissue on the surface and there would be insufficient number of neutrons reaching the tumor tissue.

In order to obtain the optimum energy of the beam, one has to use a system of filters and moderators.

The aim of our analysis was to find, using the MCNP code, what beam energy value is optimal for the therapy. 


\section{Method}

The model of the head was defined within the code as a $20-\mathrm{cm}$ diameter sphere of tissue-like material (ICRU brain) consisting of hydrogen (10.7\%), carbon (14.5\%), nitrogen $(2.2 \%)$, oxygen $(71.2 \%)$, sodium $(0.2 \%)$, phosphorus $(0.4 \%)$, sulfur $(0.2 \%)$, chlorine $(0.3 \%)$ and potassium $(0.3 \%)$ of the density of $1.04 \mathrm{~g} / \mathrm{cm}^{3}$. The sphere was divided into 60 pieces of various size: 6 layers of concentric, cylindrical cells up to the depth of $7 \mathrm{~cm}$, and the rest of the head defined as one cell (Figure 1).

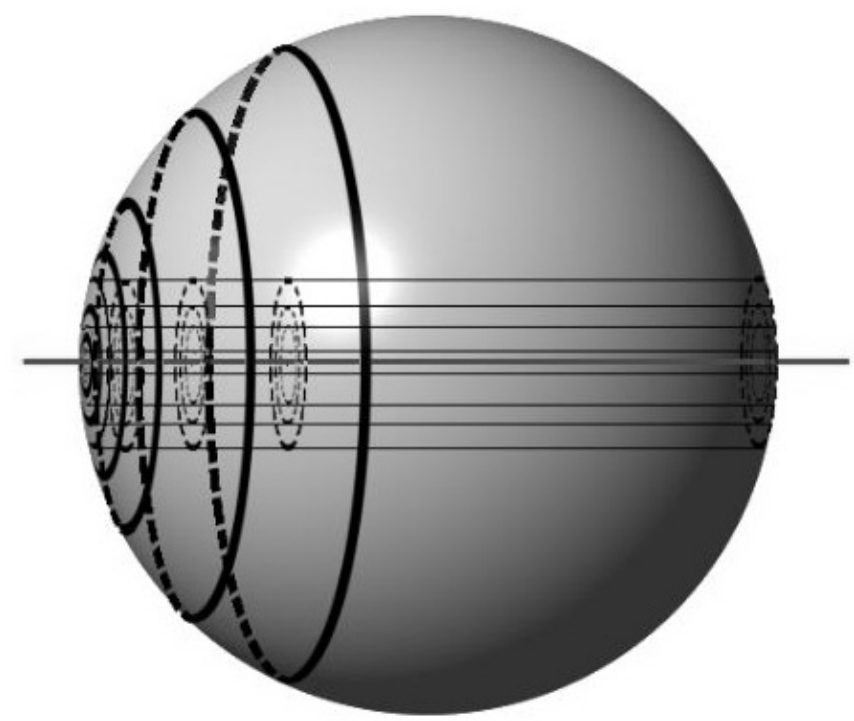

Figure 1. The model of the head used for calculations. The central line indicates the beam axis.

The energy of the neutron beam ranged between $1 \mathrm{eV}$ and $1 \mathrm{MeV}$. The beam was ideally collimated, pointing to the center of the head.

In order to study the dependence of the boron dose on the beam energy, we added isotropically ${ }^{10} \mathrm{~B}$ to the brain tissue material. The fraction matched the one used in the therapy that is $0.003 \%$, which corresponds to the mean free path of $1.5 \mathrm{~m}$. 


\section{Results and discussion}

Figure 2 shows the dependence of the absorbed boron dose on the depth and the distance from the beam axis for chosen energy values. As expected, the maximum dose depth increased with increasing beam energy. At the energy of $1 \mathrm{keV}$, the maximum dose was absorbed at the depth of $2-3 \mathrm{~cm}$, which corresponds to a typical tumor situation.
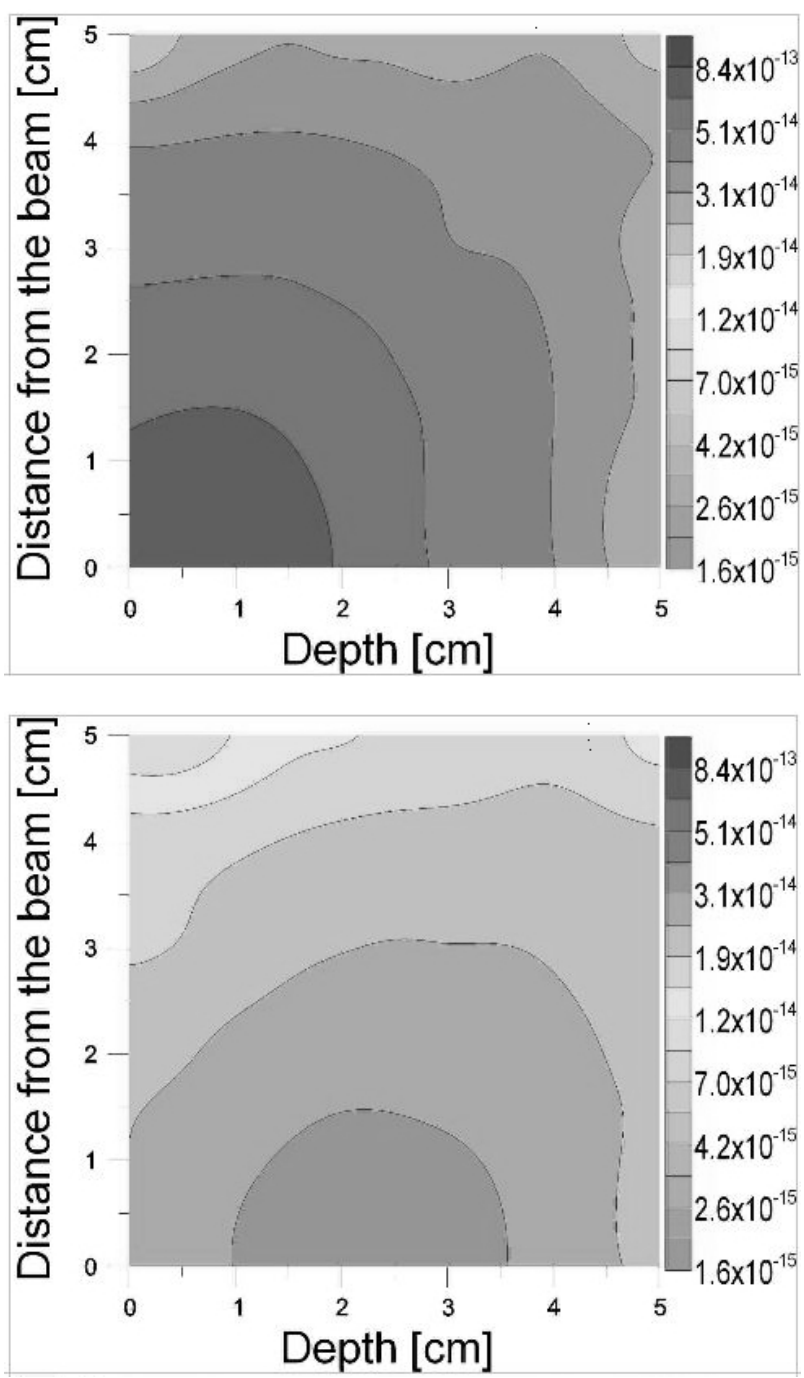


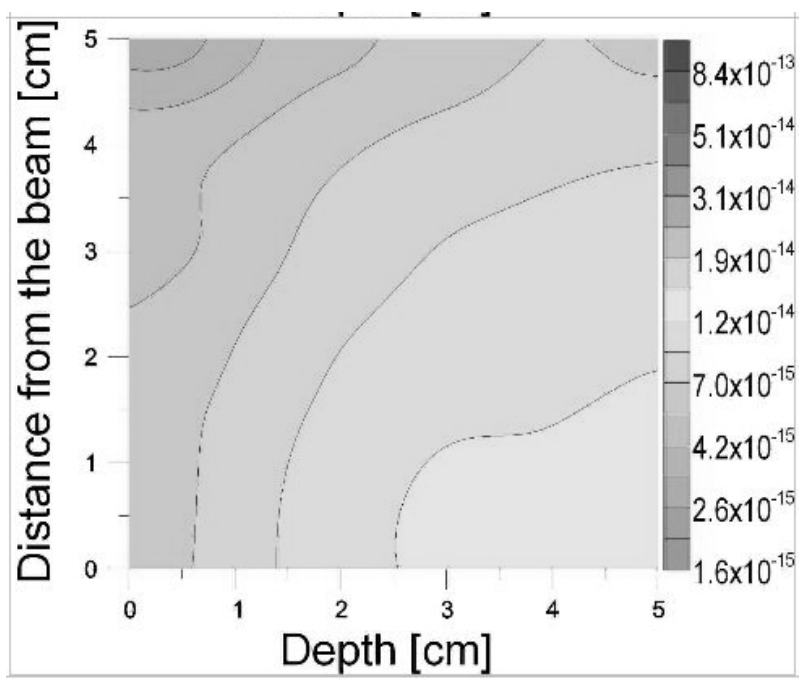

Figure 2. The dependence of the boron dose $[\mathrm{Gy} / \mathrm{n}]$ on the depth and the distance from the beam for some chosen beam energy values ( $1 \mathrm{eV}, 1 \mathrm{keV}$ and $1 \mathrm{MeV}$, respectively).

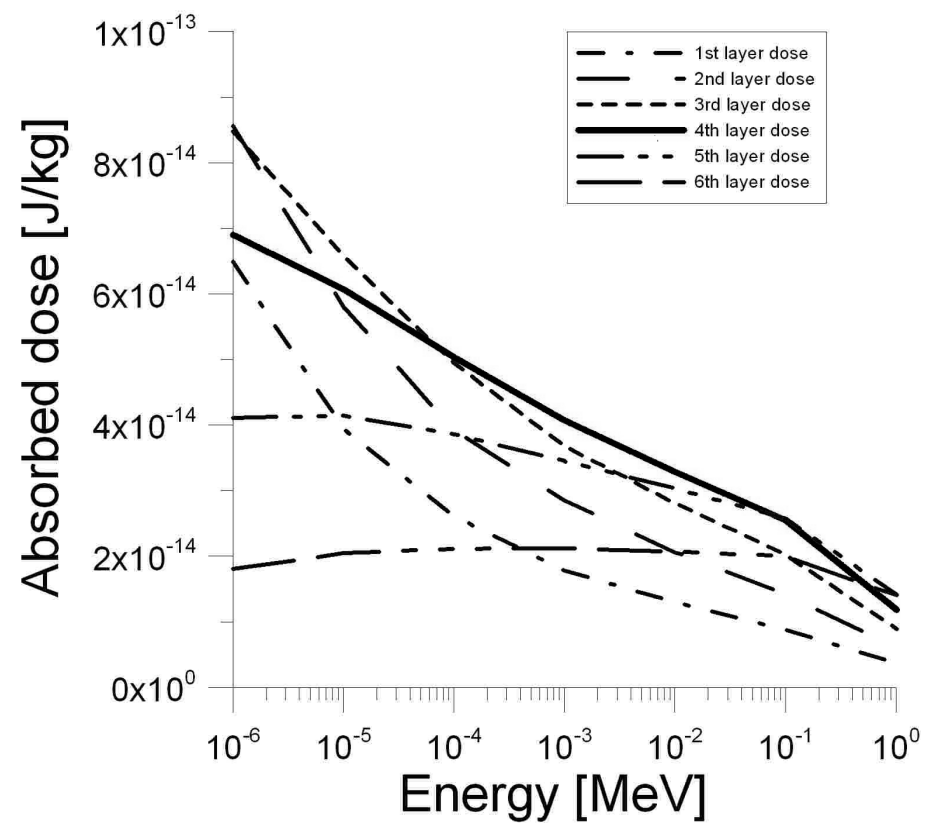

Figure 3. The dependence of the boron dose (per 1 neutron) on the beam energy for each particular layer. The thick line corresponds to the values obtained for a typical tumor depth (layer 4). 

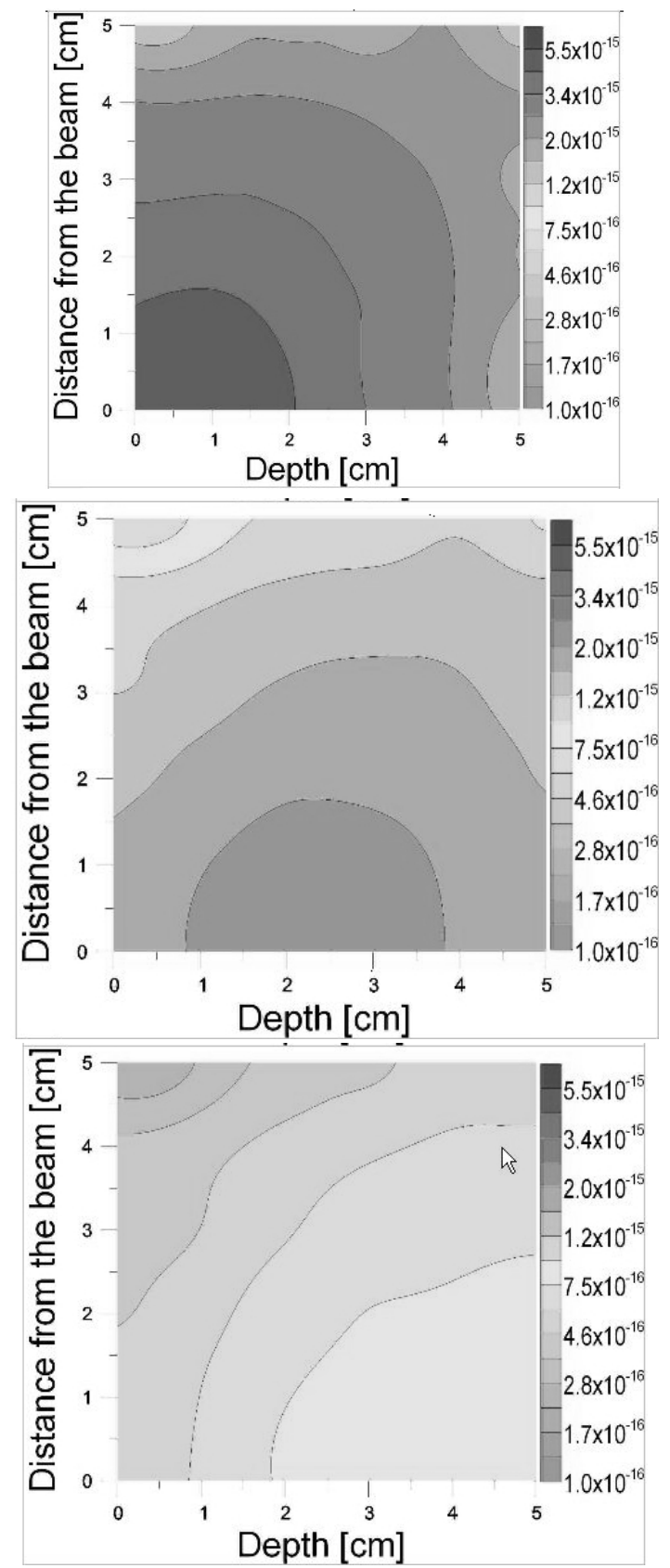

Figure 4. The dependence of the nitrogen dose $[\mathrm{Gy} / \mathrm{n}]$ on the depth and the distance from the beam for some chosen beam energy values $(1 \mathrm{eV}, 1 \mathrm{keV}$ and $1 \mathrm{MeV}$ from the top to the bottom of the figure). 
Figure 3 is a boron dose for each particular layer as a function of beam energy. For the first layers the dose decreases monotonically with the beam energy, but for deeper layers there is a maximum of the absorbed dose at the beam energies of about $1 \mathrm{keV}$.

For layer 4, corresponding to the typical tumor depth, the dependence is monotonic.

In order to study the gamma and nitrogen doses, we used ICRU brain material without the boron fraction. We made the assumption that a $0.6 \mathrm{MeV}$ proton will be stopped within the same cell it was produced (the range of $0.6 \mathrm{MeV}$ protons in tissue is $\left.1.022 \cdot 10^{-3} \mathrm{~g} / \mathrm{cm}^{2}\right)[1]$.

Figure 4 shows the dependence of the nitrogen dose on the depth and the distance from the beam for each of the energy values. Again - we obtained a strong dependence of the maximum dose depth on beam energy.

In order to obtain the value of the gamma dose, mostly from the $2.2 \mathrm{MeV}$ photons from the hydrogen capture reaction, we have calculated the energy deposited by photons in each cell and divided it by the mass of the cell.

Due to the properties of gamma radiation, the shape of the gamma dose dependence on the beam energy differs from that for other dose components under study.

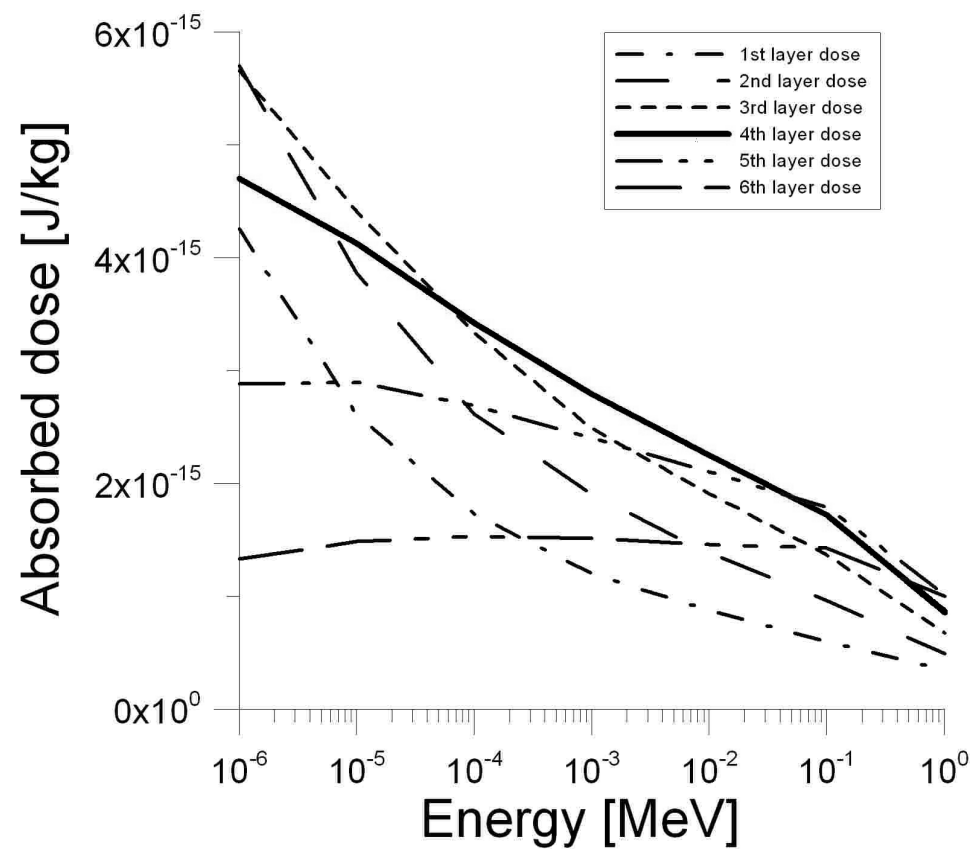

Figure 5. The dependence of the nitrogen dose on the beam energy for each particular layer.

The thick line corresponds to the values obtained for a typical tumor depth (layer 4). 

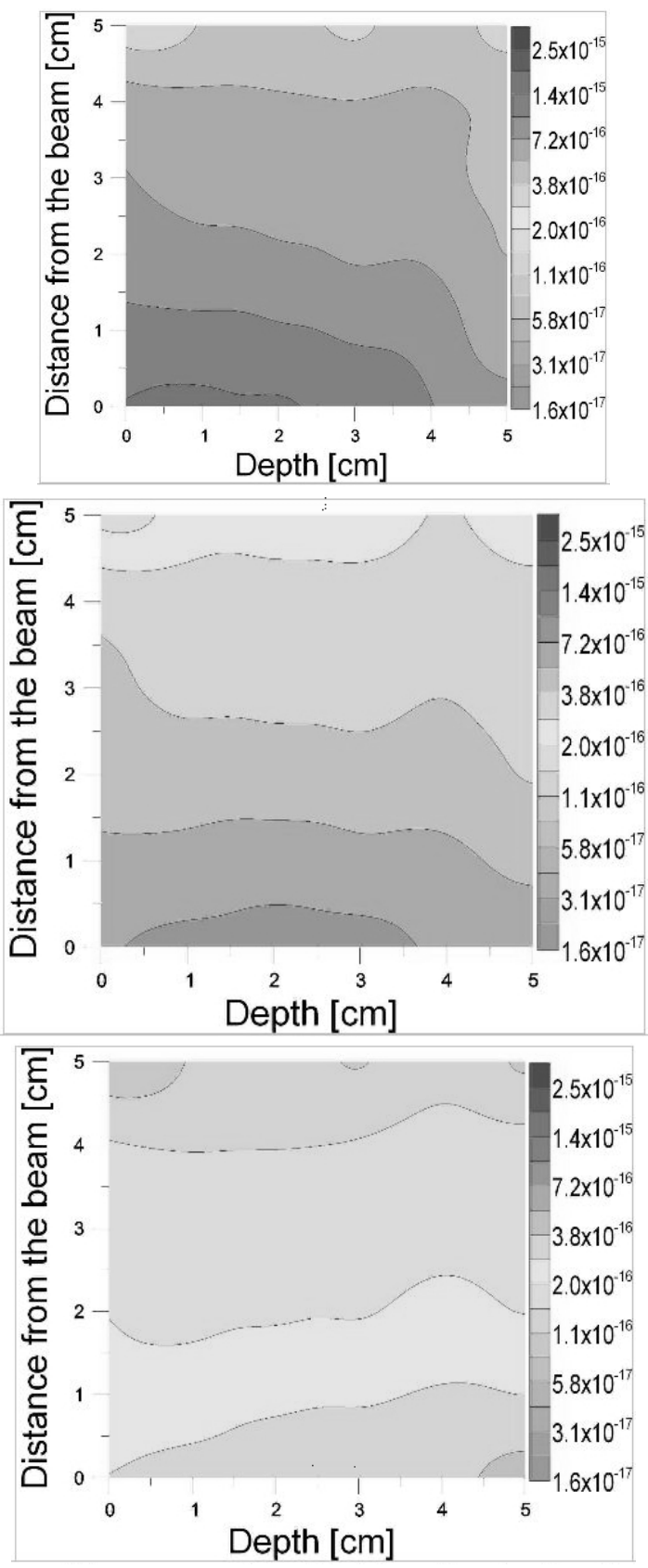

Figure 6. The dependence of the gamma dose $[\mathrm{Gy} / \mathrm{n}]$ on the depth and the distance from the beam for some chosen beam energy values ( $1 \mathrm{eV}, 1 \mathrm{keV}$ and $1 \mathrm{MeV}$ respectively). 


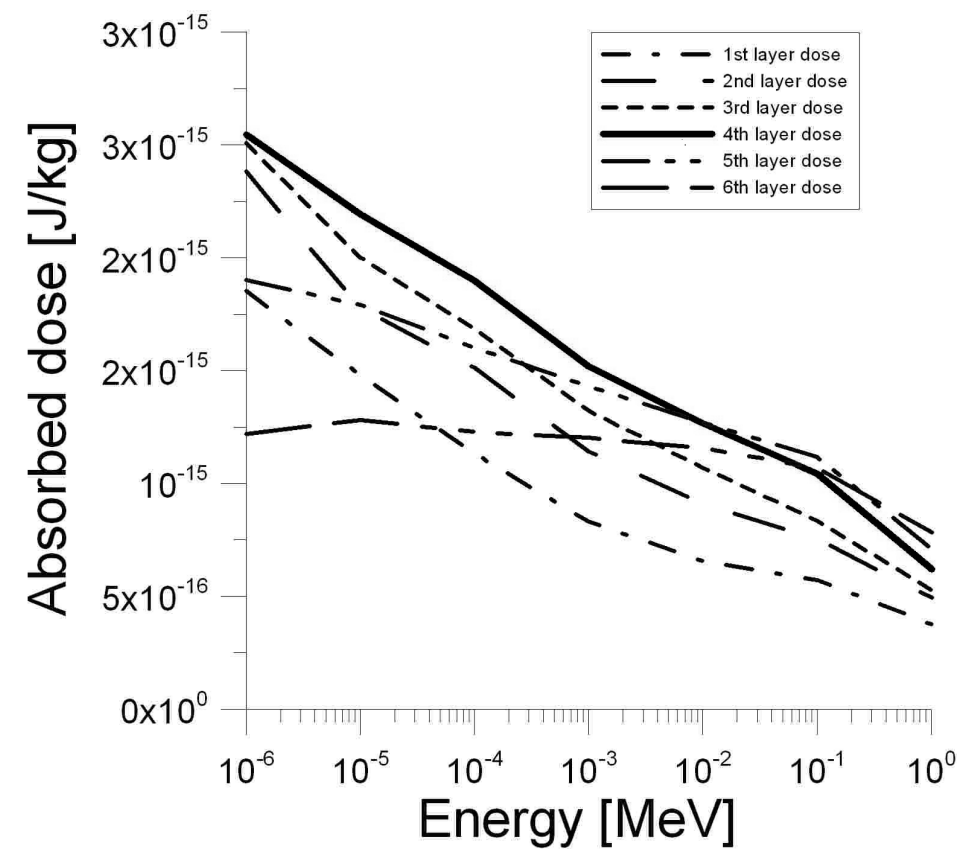

Figure 7. The dependence of the gamma dose on the beam energy for each particular layer. The thick line corresponds to the values obtained for a typical tumor depth (layer 4).

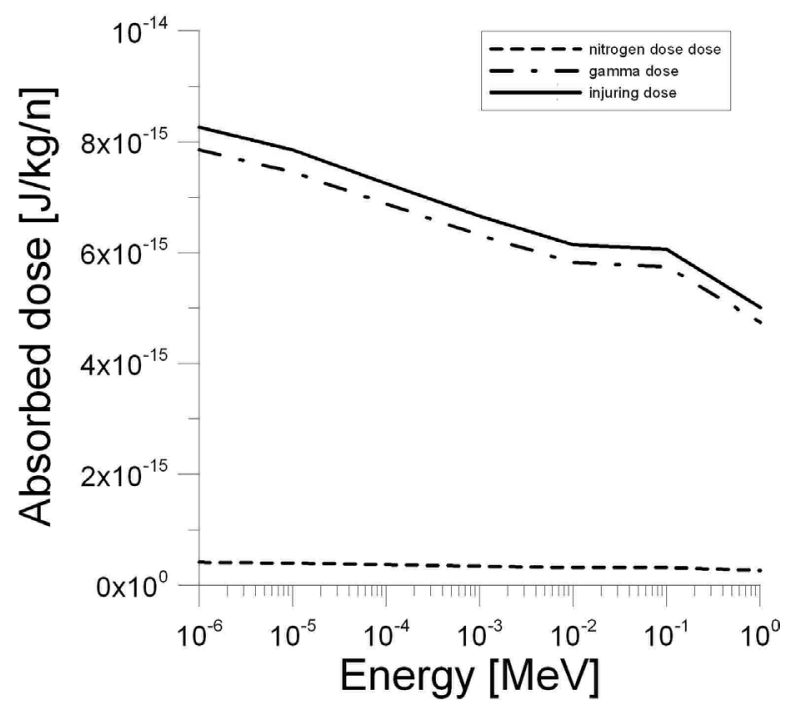

Figure 8. The gamma and nitrogen doses and their sum, averaged over the whole head. 


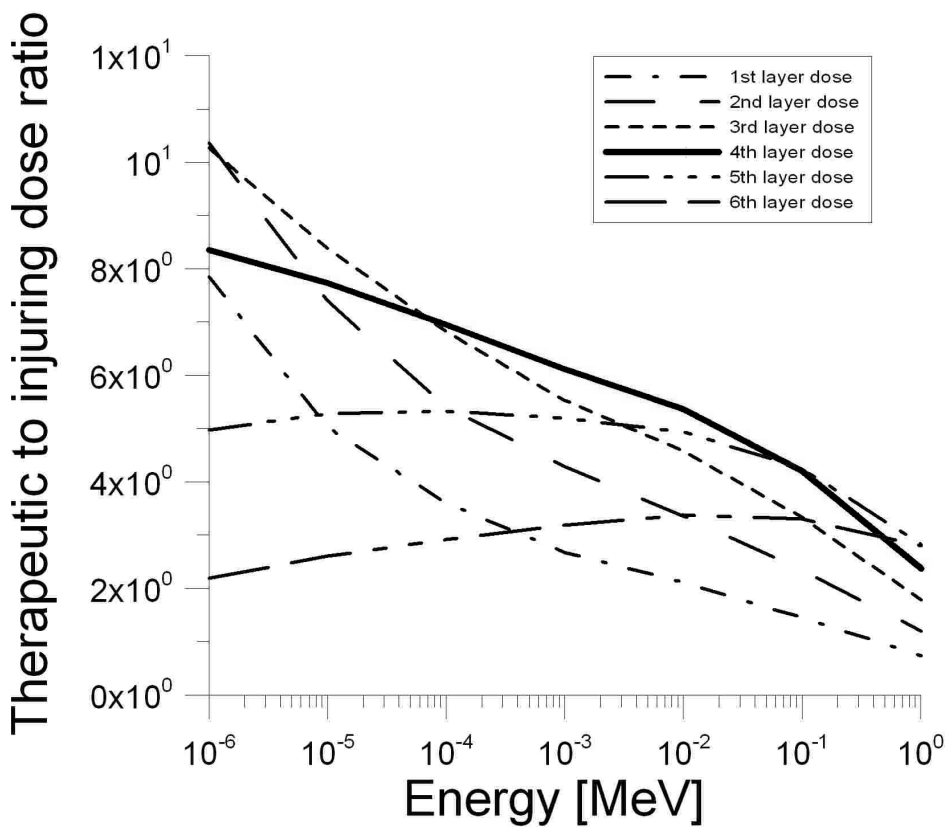

Figure 9. The ratio of the therapeutic dose absorbed in each particular layer to the injuring dose averaged over the patient's whole head. The thick line corresponds to the values obtained for a typical tumor depth (layer 4).

Like in those other components, the dose for the first layers decreases monotonically as a function of beam energy, but for the deepest layer there is a maximum dose absorbed at the beam energy of $1 \mathrm{keV}$.

In order to interpret these results correctly, one should realize that in the therapy the boron dose will be absorbed only in some selected (tumor) area, whereas the gamma and nitrogen doses will be absorbed in the patient's whole head. The average nitrogen and gamma injuring doses and their sum are presented in Figure 8. One can see that for the whole head the gamma dose is one order of magnitude higher than the nitrogen dose, while the values obtained for the layers investigated were similar.

In order to determine the optimum beam energy range, one should compare the average injuring dose to the therapeutic dose absorbed in each of the layers. The result of this comparison is presented in Figure 9. 


\section{Conclusions}

Our results will be used to find an optimum beam energy range. For a more complete picture, the calculations concerning the fast neutron dose and the gamma dose from the beam should be taken into account. This will be done as the next step in our study.

\section{Acknowledgements}

The work was supported by the Polish Ministry of Sciences and Higher Education, under grant no. PBZ/MEiN/01/2006/43.

\section{References}

[1] http://physics.nist.gov/PhysRefData/Star/Text/contents.html 